\title{
ON THE PCR POLICING OF AN ARBITRARY STREAM PASSING THROUGH A PRIVATE ATM NETWORK BEFORE REACHING THE PUBLIC UNI
}

\author{
Lorenzo Battaglia \\ Digital Communication Systems Dept. \\ Technical University of Hamburg-Harburg \\ Germany \\ Battaglia@TU-Harburg.de \\ Ulrich Killat \\ Digital Communication Systems Dept. \\ Technical University of Hamburg-Harburg \\ Germany \\ Killat@TU-Harburg.de
}

\begin{abstract}
Any Call Admission Control algorithm and any Network Dimensioning depend on the reliability of the chosen Usage Parameter Control algorithm. The standardized ATM UPC algorithm is the GCRA. An ideal ATM PCR policer should guarantee a very low cell loss probability $\left(10^{-9}\right)$ for the cells of a well-behaved user and police contract violation by the source as rigidly as possible. All this independently of the characteristics of the monitored stream and of the background traffic. When we started our investigations no acceptable GCRA parameters dimensioning had been presented and no alternative policing solution could claim to be more general and simpler than the GCRA. We already provided an analytic GCRA parameters dimensioning which transforms the GCRA into an ideal PCR policer, also in the case of online PCR renegotiation, and into an ideal ACR policer of ABR services. In this paper we generalize our results to the PCR policing of a GCRA $\left(T_{P C R} ; \tau_{0}\right)$ complying stream passing through an ATM network before reaching the public UNI. The GCRA dimensioning we present allows the policer to discard no cell of a well-behaved user and to rigidly police contract violation. Our results hold for any kind of background traffic.
\end{abstract}

Keywords: $\quad$ ATM, Traffic Monitoring, Network Dimensioning 


\section{INTRODUCTION}

ATM is the switching and multiplexing technology chosen by the ITU for the operation of B-ISDN. Basically, ATM technology is designed to combine the reliability of circuit switching with the efficiency and flexibility of packet switching technologies [1]. ATM provides 5 service categories with a rich set of related QoS parameters and guarantees an end-to-end QoS to individual connections [3].

Call Admission Control (CAC), Network Dimensioning and Usage Parameter Control (UPC) are very important issues in ATM technology. They all depend on how input traffic is described, on how cell streams' characteristics change when passing an ATM queueing system. CAC and Network Dimensioning strongly depend on how reliable the chosen UPC algorithm is. The standardized ATM UPC algorithm is the Generic Cell Rate Algorithm (GCRA). The GCRA depends on two parameters: the Increment $I$ and the Limit $L$. When just one GCRA is used, Peak Cell Rate (PCR) policing, I and L are respectively denoted by $T$ and $\tau$. If two GCRA's are used, PCR and Sustainable Cell Rate (SCR) policing, the four parameters are respectively denoted by $T, \tau, T_{s}$ and $\tau_{s}$. The ATM Forum has established that streams at the input of ATM networks should be described by means of the GCRA parameters used to police them. A recent internet draft on Differentiated Services [14] has proposed to assign drop precedence levels of packets within the same Assured Forwarding class by using a leaky bucket policer. Hence the GCRA concept has received increasing attention for both, ATM-based and IP-based networks.

One of the main criteria to dimension an ATM policer is to minimize cell loss. The loss of a single ATM cell would cause the loss of a higher layer's packet, resulting in wasted bandwidth and loss of QoS. At the same time it is very important for the network provider that any malicious user is policed as rigidly as possible. Any charging system as well as any network dimensioning to guarantee a desired QoS is valid only if all cell streams entering the network do comply with the traffic contract. An ideal ATM policer should then guarantee a very low cell loss probability $\left(10^{-9}\right)$ for the cells of a well-behaved user and police contract violation as rigidly as possible. All this independently of the characteristics of the monitored stream and of the cell delay variations due to the other ATM applications, whose cells pass through the same ATM queueing systems (see ATM UPC definitions in [3] and Figure 4-2 therein). To be able to protect all network resources, the policing function must be located as close as possible to the actual traffic source [20] and obviously be under direct control of the network provider. Since this function must be available for every connection during the entire active phase and must operate in real time, the policing method used must be fast, simple and cost effective. 
Since the dimensioning of GCRA parameters was left to the user without clear guidelines, several alternative policing methods have been introduced in the literature. Most of them have been evaluated against their capability to enforce the PCR of ON-OFF sources. All have been compared to the GCRA. Window-based control methods have been proposed [12], [Rathgeb91]. Fuzzy Logic [10]-[13], Neural Networks [22] and Finite State Automata [18] control methods have been presented. Even "improved" versions of the GCRA [2], [ 4], [16], [17] have been tested. No solution could however claim to be more general and simpler than the GCRA.

Many other authors investigated the rejection probability $p_{r}$ of the GCRA when monitoring a CBR source passing an ATM multiplexer. Some, e.g. [15], tried to relate the statistical parameters of the background traffic to the chosen GCRA parameters. Others, e.g. [9], saw the whole as a $(M+D) / D / 1$, FIFO. All came to the conclusion that in order to ensure a sufficiently low value for $p_{r}$ it is necessary to police a higher rate than negotiated, in some cases [8] even significantly higher. Such a choice is surely not the ideal to rigidly police contract violation by the source. We started our investigations with the task to dimension the GCRA in such way to minimize the rejection probability for the cells of a well-behaved user and to rigidly police contract violation by the source, and this independently of the characteristics of the monitored stream and of the background traffic. We started with the rejection probability of a GCRA when monitoring a well-behaved CBR stream passing an ATM multiplexer. We extended our results also to the more general case of an ATM multiplexer equipped with a global traffic shaper which guarantees a minimum distance of $T_{\text {shape }}$ time slots between two consecutive cells [5]. We treated then the PCR policing of any stream passing an ATM multiplexer, also in the case of on-line PCR renegotiation, and the Available Cell Rate (ACR) policing of ABR services [6]. In [7] we dimensioned two sets of GCRA parameters in such way to fairly police PCR and SCR of an arbitrary stream passing through an ATM multiplexer. In this paper we treat the PCR policing of an arbitrary stream which complies with a $G C R A\left(T_{P C R}, \tau_{0}\right)$ passing through a private ATM network before reaching the public User Network Interface (UNI), where the policing function is performed. According to the PCR Reference Model [ 3] we refer to a "private" and to a "public" network to distinguish two networks with different providers. Also in this case we provide a GCRA parameters dimensioning which transforms the GCRA into an ideal PCR policer: No cell of a well-behaved user is discarded whereas contract violation by the source is policed as rigidly as possible. Our results hold for any kind of background traffic (even self similar) generated by the other ATM applications running at the user side, whose cells pass through the same ATM queueing systems in the private ATM network. This paper is organized as follows. In Section 1 we 
model the problem. In Section 2 some important concepts of the GCRA are recalled. Section 3 provides the framework for GCRA parameters dimensioning. In Section 4 and 5 the policing issue respectively of streams complying with a $G C R A\left(T_{P C R}, 0\right)$ and with a $G C R A\left(T_{P C R}, \tau_{0}\right)$ is treated.

\section{MODELLING THE PROBLEM}

We treat the PCR policing of an arbitrary stream passing through a private network before reaching the public UNI. We refer to Fig.2. Let $S$ be the source we want to monitor. The monitored stream passes through $M$ queues (multiplexers and switches) before entering the public ATM UNI. We assume each queue, e.g. the $k^{t h}$, to have $N_{k}$ input lines and one output link and to be equipped with a buffer of finite length $B_{k}$ (see also example in Fig. 3). Time slots on each of the input lines and on the output link are of the same length and synchronized to each other. Every new cell is assumed to arrive at the beginning of a slot and can be transmitted at the end of the same time slot if no predecessor exists (see Fig.1).

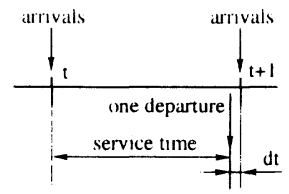

Figure 1 Queue Discipline: Departure occurs immediately before the beginning of the next time slot.

About the background traffic and the topology of the rest of the network we don't make any hypotheses. This choice may at first glance be surprising

Private ATM Network

Public UN

Public ATM Network
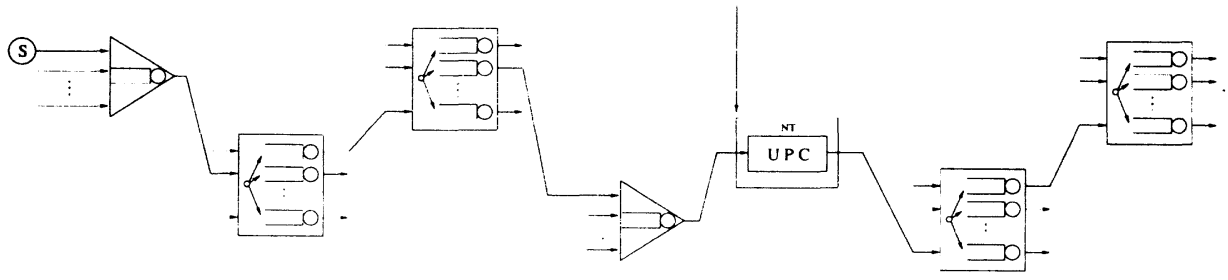

Figure 2

because the delay behaviour of the monitored cells will, of course, depend on the characteristics of the background traffic and on the topology of the rest of 
the network. However, since $B_{k}$ time slots is the upper-bound to the delay any monitored cell can experience passing through an ATM queueing system equipped with a buffer of length $B_{k}$ and a server, it is possible to provide an ideal GCRA dimensioning once the negotiated PCR and the lengths of the crossed buffers $\left\{B_{k}: k=1, \ldots, M\right\}$ are known. In the ensuing discussion we denote by $G C R A(T, \tau)$-stream $(G C R A(T, \tau)$-source) an arbitrary stream (source) which complies with a $G C R A(T, \tau)$.

\section{THE GENERIC CELL RATE ALGORITHM}

The GCRA depends on two parameters the Increment, $I$, and the Limit, $L$. In order to get familiar with it we refer to a $G C R A(I, L)$. Let's consider a possible realization of the departure process of a monitored stream from the last ATM queueing system. Let $t_{a}(i)$ be the discrete time instant in which the $i^{\text {th }}$ cell of the monitored stream arrives at the policer and $T A T(i)$ its Theoretical Arrival Time. Whenever the $i^{\text {th }}$ cell arrives after its TAT, i.e. $t_{a}(i)>T A T(i)$, it is accepted and the following $T A T$ is given by

$$
T A T(i+1)=t_{a}(i)+I .
$$

Else it is accepted only if

$$
T A T(i)-L \leq t_{a}(i)
$$

holds and the new $T A T$ is given by

$$
T A T(i+1)=T A T(i)+I .
$$

\section{HOW TO TUNE $I$ AND $L$}

In order to rigidly police contract violation by the source we set $I$ to the reciprocal of the negotiated rate. In order to reject no cell of a well-behaved user for any background traffic, i.e. to properly dimension $L$, we introduce the new concept of $\tau$ coverage area (Subsection 3.1) and apply it to the worst case sequence into which the monitored stream can be transformed by the background traffic (Subsection 3.2). Since for the PCR policing $L$ is denoted by $\tau$, in the ensuing discussion we coherently refer to $\tau$.

\subsection{THE $\tau$ COVERAGE AREA}

Any stream of $m$ cells arriving at the GCRA is uniquely identified by the sequence of the discrete time instants $t_{a}(i), i=1,2, \ldots, m$, in which the $i^{\text {th }}$ monitored cell enters the policer. Let TAT(i), $i=2,3, \ldots, m$, be the corresponding sequence of the Theoretical Arrival Time of the $i^{\text {th }}$ cell at the GCRA. Whenever the $i^{\text {th }}$ cell arrives at the policer before its TAT any

$$
\tau \geq T A T(i)-t_{a}(i)
$$


allows to accept that cell. We define $\tau$ coverage area, $\tau_{C A}$, for the $i^{\text {th }}$ cell as

$$
\tau_{C A}(i)=\left[T A T(i)-t_{a}(i)\right]^{+}
$$

i.e. the smallest value of $\tau$ that allows the policer to accept it. Obviously a $\tau_{C A}$ of 0 time slots is already enough to accept any cell arriving after its TAT. Considering the whole sequence and defining

$$
\tau_{C A \max }=\max \left\{\tau_{C A}(2), \tau_{C A}(3), \ldots, \tau_{C A}(m)\right\}
$$

any $\tau \geq \tau_{C A \max }$ allows then to accept all the cells without losses. Such an a posteriori dimensioning of $\tau$ will be valid for any sequence $t_{a}(i), i=1,2, \ldots$, if derived from the worst case sequence into which the monitored stream can be transformed by the background traffic.

\subsection{THE WORST CASE SEQUENCE}

We define as the worst case sequence into which the monitored stream passing through a private ATM network can be transformed by the background traffic the sequence $t_{a}(i), i=1,2, \ldots$, for which each $\tau_{C A}(i), i=2,3, \ldots$, is the biggest possible. Whenever the $i^{t h}$ monitored cell enters the policer before its TAT

$$
\tau_{C A}(i)=T A T(i)-t_{a}(i)
$$

holds. The biggest $\tau_{C^{\prime} A}(i)$ will then occur when $T A T(i)$ and $t_{a}(i)$ are respectively the biggest and the smallest allowed for $i$. We denote the latter quantity by $t_{a \min }(i)$. To simplify the results, in the ensuing discussion we denote as time slot 0 that in which the first of the monitored cells enters the first queue of the private ATM network. Moreover, since we are interested in the difference $T A T(i)-t_{a}(i)$ we don't consider in the delay the service time (1 time slot) which all cells experience passing through each ATM server. Since with the previous assumption the delay that any monitored cell will experience passing through all the $M$ queues is at most given by

$$
\sum_{k=1}^{M} B_{k}
$$

time slots, the biggest TAT allowed for $i$ is

$$
\operatorname{TAT}(i)=\sum_{k=1}^{M} B_{k}+(i-1) I .
$$

We assume lines to be short and routing operations to be performed fast enough not to introduce any further delay. As far as the $t_{a \min }(i)$ is concerned, 
it is the first time slot in which the $i^{\text {th }}$ monitored cell can at the earliest leave the $M^{\text {th }}$ queue. Formally

$$
t_{a \min }(i)=\max \left\{t_{a Q}(i, M), t_{a \min }(i-1)+1\right\}
$$

with

$$
t_{a Q}(i, j)=\max \left\{t_{a Q}(i, j-1), t_{a Q}(i-1, j)+1\right\}
$$

and $j=1,2, \ldots, M$. We denote by $t_{a Q}(i, j)$ the earliest arrival time of the $i^{t h}$ monitored cell at the $j^{\text {th }}$ queue and by $t_{a Q}(i, 0)$ the earliest departure instant of the $i^{\text {th }}$ cell from the monitored source. Clearly $t_{a \text { min }}(i)$, as in general $t_{a Q}(i, j)$ is the latest of the two events: the earliest arrival time of the $i^{t h}$ cell at the previous queue (if the $(i-1)^{t h}$ monitored cell has already left the previous queue, the $i^{\text {th }}$ monitored cell can leave it at the earliest immediately) or right after the departure of the $(i-1)^{t h}$ monitored cell from the previous queue (if another previous monitored cell is in the previous queue, the $i^{\text {th }}$ monitored cell can leave it at the earliest right after the $\left.(i-1)^{t h}\right)$. The worst case sequence occurs then when the background traffic delays the first of the monitored cells by $\sum_{k=1}^{M} B_{k}$ time slots without delaying any of the following ones. The $\tau_{C A}$ sequence is then in the worst case given by

$$
\tau_{C A}(i)=\sum_{k=1}^{M} B_{k}+(i-1) I-t_{a \min }(i)
$$

with $i=2,3, \ldots$. In order to rigidly police contract violation by the source we set $I$ to the reciprocal of the negotiated rate. We will show that for the worst case sequence the biggest $\tau_{C A}(i), i=2,3, \ldots$, is a finite quantity. We consistently denote the last quantity by $\tau_{C A \max }$.

This $\tau_{C A \max }$ will then be the ideal $\tau$ to police the PCR of any stream, passing through a private ATM network guaranteeing that for any background traffic no cell of a well-behaved user will be rejected. Any smaller $\tau$ would lead to a positive probability to reject a cell of a well-behaved user. Any bigger $\tau$ would tolerate cell delay variations which wouldn't ever occur in the stream of a well-behaved user.

\section{PCR POLICING ISSUE OF A $G C R A\left(T_{P C R}, 0\right)-$ -STREAM PASSING THROUGH A PRIVATE ATM NETWORK}

We consider the PCR policing of a $G C R A\left(T_{P C R}, 0\right)$-stream passing through $M$ queues at the user's premises before reaching the public UNI (see example in Fig.3). Passing through the $M$ queues the monitored cells will in general experience cell delay variations that can make appear the monitored source to 
send at a higher rate than negotiated. We use a $G C R A(T, \tau)$ to police this stream. Since in the worst case any $G C R A\left(T_{P C R}, 0\right)$-source is a well-behaved CBR source with period $T_{P C R}$, GCRA parameters can a fortiori be dimensioned so to fairly police such a source. To rigidly police contract violation by the source we set $T=T_{P C R}$. To evaluate the $\tau_{C A \max }$ two cases have to be considered: $\sum_{k=1}^{M} B_{k}<T_{P C R}$ and $\sum_{k=1}^{M} B_{k} \geq T_{P C R}$.

\subsection{FIRST CASE: $\sum_{K=1}^{M} B_{K}<T_{P C R}$}

In this case, since no cell can be delayed of more time slots than its period

$$
t_{a \min }(i)=t_{a Q}(i, M)=t_{a Q}(i, M-1)=\ldots=t_{a Q}(i, 0)
$$

holds. Since

$$
t_{a Q}(\imath .0)=(i-1) T_{P C R}
$$

then

$$
\tau_{C A}(i)=\sum_{k=1}^{M} B_{k}+(i-1) T_{P C R}-(i-1) T_{P C R}=\sum_{k=1}^{M} B_{k}
$$

with $i=2,3, \ldots$, for the worst case sequence holds. Clearly $\tau_{C A m a x}=$ $\sum_{k=1}^{M} B_{k}$.

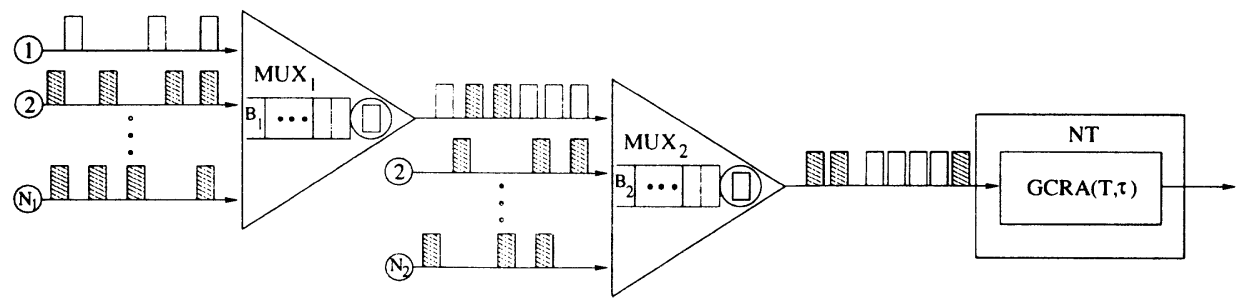

Figure 3 The PCR policing issue of a $G C R A\left(T_{P C R}, \tau_{0}\right)$-stream feeding input 1 of the first multiplexer: The background traffic can make appear the monitored source to send at a higher rate than negotiated.

\subsection{SECOND CASE: $\sum_{K=1}^{M} B_{K} \geq T_{P C R}$}

The worst case sequence has at first a burst of

$$
b=\left\lfloor\frac{\sum_{k=1}^{M} B_{k}}{T_{P C R}-1}\right\rfloor+1
$$


cells spaced at one time slot, followed by all the other cells which don't experience any delay. The $\tau_{C A}$ sequence is then

$$
\tau_{C A}(i)=\left\{\begin{aligned}
(i-1)\left(T_{P C R}-1\right) & \text { for } 2 \leq i \leq b \\
\sum_{k=1}^{M} B_{k} & \text { for } i>b
\end{aligned}\right.
$$

since in this case

$$
t_{a \min }(i)=\left\{\begin{aligned}
i+\sum_{k=1}^{M} B_{k}-1 & \text { for } 2 \leq i \leq b \\
t_{a Q}(i, 0) & \text { for } i>b
\end{aligned}\right.
$$

Thus

$$
\tau_{C A \max }=\max \left\{\tau_{C A}(2), \tau_{C A}(3), \ldots, \tau_{C A}(b), \sum_{k=1}^{M} B_{k}\right\} .
$$

Since the sequence $\tau_{C A}(2), \ldots, \tau_{C A}(b)$ is monotonically increasing and

$$
\tau_{C A}(b) \leq \sum_{k=1}^{M} B_{k}
$$

$\tau_{C \text { Amax }}=\sum_{k=1}^{M} B_{k}$. Hence setting $T$ to $T_{P C R}$ and choosing $\tau=\sum_{k=1}^{M} B_{k}$ allows to ideally police the PCR of any stream passing through $M$ buffers having lengths $\left\{B_{k}: k=1, \ldots, M\right\}$, so that for any background traffic contract violation by the source is rigidly policed and so that no cell of a well-behaved user is discarded.

\section{PCR POLICING ISSUE OF A $G C R A\left(T_{P C R}, \tau_{0}\right)-$ -STREAM PASSING THROUGH A PRIVATE ATM NETWORK}

We consider now the PCR policing of a $G C R A\left(T_{P C R}, \tau_{0}\right)$-stream passing through $M$ queues at the user's premises before reaching the public UNI. We use a $\operatorname{GCRA}(T, \tau)$ to police this stream. We analyze at first the case of $M=1$ and then generalise our results. In order to rigidly police contract violation by the source we set $T=T_{P C R}$. In order to dimension $\tau$ we evaluate the $\tau_{C A m a x}$. Clearly each $\tau_{C A}(i), i=2,3, \ldots$, is the biggest possible if the first monitored cell of the $G C R A\left(T_{P C R}, \tau_{0}\right)$-stream experiences the longest possible delay in the queue before being served and if the following cells arrive at the earliest (see definition of the $t_{a Q}(i, 0)$ ) and are not delayed by any further background traffic. We turn now our attention to each $t_{a Q}(i, 0), i=1,2, \ldots$, which we already defined as the earliest departure instant of the $i^{t h}$ cell from the monitored source. Two cases have to be considered: $\tau_{0}<T_{P C R}$ and $\tau_{0} \geq T_{P C R}$. 


\subsection{CASE OF $\tau_{0}<T_{P C R}$}

In this case

$$
t_{a Q}(i, 0)=\left\{\begin{aligned}
0 & \text { for } i=1 \\
(i-1) T_{P C R}-\tau_{0} & \text { for } i \geq 2
\end{aligned}\right.
$$

\subsection{CASE OF $\tau_{0} \geq T_{P C R}$}

In this case there's at first a burst of $N$ back-to-back cells with

$$
N=\left\lfloor\frac{\tau_{0}}{T_{P C R}-1}\right\rfloor+1
$$

and

$$
t_{a Q}(i, 0)=\left\{\begin{aligned}
i-1 & \text { for } i=1,2, \ldots, N \\
(i-1) T_{P C R}-\tau_{0} & \text { for } i \geq N+1
\end{aligned}\right.
$$

The issue we address can be simply solved by means of an equivalence. Let's consider the PCR monitoring of a well-behaved source passing through an ATM queueing system equipped with a buffer of length $B$. Let $T_{P C R}=1 /$ PCR. For the worst case sequence into which such a stream can be transformed by the background traffic [5] two cases have to be considered: $B<T_{P C R}$ and $B \geq T_{P C R}$.

\subsection{CASE OF $B<T_{P C R}$}

In this case

$$
t_{a Q}(i, 0)=\left\{\begin{aligned}
0 & \text { for } i=1 \\
(i-1) T_{P C R}-B & \text { for } i \geq 2 .
\end{aligned}\right.
$$

\subsection{CASE OF $B \geq T_{P C R}$}

In this case there's at first a burst of $b$ back-to-back cells with

$$
b=\left\lfloor\frac{B}{T_{P C R}-1}\right\rfloor+1
$$

and

$$
t_{a Q}(i, 0)=\left\{\begin{aligned}
i-1 & \text { for } i=1,2, \ldots, b \\
(i-1) T_{P C R}-B & \text { for } i \geq b+1 .
\end{aligned}\right.
$$

The PCR monitoring of a $G C R A\left(T_{P C R}, \tau_{0}\right)$-stream is then equivalent to the PCR monitoring of a $G C R A\left(T_{P C R}, 0\right)$-source, whose cells pass through an ATM queueing system equipped with a buffer of length $\tau_{0}$. The results of the 
previous Section can be then generalized to this case. Setting $T$ to $T_{P C R}$ and choosing

$$
\tau=\sum_{k=1}^{M} B_{k}+\tau_{0}
$$

allows to ideally police the PCR of any $G C R A\left(T_{P C R}, \tau_{0}\right)$-stream passing through $M$ buffers having lengths $\left\{B_{k}: k=1, \ldots, M\right\}$ so that for any background traffic contract violation by the source is rigidly policed and so that no cell of a well-behaved user is discarded.

\section{Discussion}

We have presented a worst-case GCRA parameters dimensioning. Setting $T$ to $T_{P C R}$ and $\tau$ to $\tau_{C A m a x}$, the maximum cell delay variation a cell can experience when passing through an ATM queueing system, allows the GCRA to rigidly police contract violation by the source and to discard no cell of a well-behaved user. All this for any kind of background traffic. Any smaller $\tau$ would lead to a positive probability to reject cells of a well-behaved user. At the same time a smaller $\tau$ would decrease the potential cell clumping allowed at the UNI. The biggest cell-burst a source policed by a $G C R A(T, \tau)$ is allowed to send at a (higher) rate $1 / \delta$ (cells/time slot) is of

$$
N=\left\lfloor 1+\frac{\tau}{T-\delta}\right\rfloor
$$

cells. In order to achieve a smaller $N$, it could be thought to choose by means of simulations a $\tau<\tau_{C A \max }$, which still guarantees a sufficiently low discard probability to the cells of a well-behaved user. The main question is then if this is always necessary. If it is possible in some cases to take advantage of this general solution. Simulations have big disadvantages. They are time consuming (even with speed-up algorithms) and, above all, very complex models are needed to simulate "real" traffic. According to Eq. 21 the potential cell clumping becomes less significative the smaller $\tau$ is with respect to $T$. According to real buffer lengths, to real network speeds and to the real rate requirements of the most used applications, $\tau_{C A \max }$ comes out to be far smaller than $T$ so that in most of the cases no further optimization of $\tau$ is necessary.

\section{Conclusions}

In this paper we have addressed the PCR policing issue of a stream which complies with a $G C R A\left(T_{P C R}, \tau_{0}\right)$ passing through a private ATM network before reaching the public UNI.

We have provided an ideal dimensioning of GCRA parameters which transforms the GCRA into an ideal PCR policer. Independently of the characteristics 
of the monitored stream and of the background traffic, no cells of a well-behaved user will be discarded, whereas any malicious user will be policed as rigidly as possible.

With our policing solution no well-behaved user will experience loss of QoS due to the unjustified discard of an ATM cell. Any CAC and any charging system will work and any network dimensioning will be valid since cell streams allowed to enter the ATM network will comply with the traffic contract.

\section{References}

[1] R. Ahmad, Next Generation of Broadband Network Architectures-Traffic Flow Control and End-to-End Performance, IFIP ATM '98, 20th-22nd July 1998, Craiglands Hotel, Ilkley, UK.

[2] A. Atlasis, G. Stassinopulos, A. Vasilakos, Leaky bucket mechanism with learning algorithm for ATM traffic policing, IEEE Symp. on Comp. and Commun. - Proc. 1997. IEEE Los Alamitos, CA, pp 68-72

[3] ATM Forum, Traffic Management Specification, Version 4.0, af-tm-0056.000, April 1996.

[4] W. Bao, S. Cheng, Parameter adaptation mechanism for ATM traffic policing, Chinese Journal of Electronics, vol. 6, no. 1, Jan. 1997.

[5] L. Battaglia, Z. Bažanowski, U. Killat Dimensioning GCRA Parameters to Fairly Police a CBR Stream Passing an ATM Multiplexer Equipped with a Traffic Shaper, IFIP ATM '98, 20th-22nd July 1998, Craiglands Hotel, Ilkley, UK.

[6] L. Battaglia, U. Killat QoS of IP over ATM: The PCR and ACR Policing Issue, IWS '99, 18th-20th Feb. 1999, Osaka University Convention Center, Suita, Japan.

[7] L. Battaglia, U. Killat How to Dimension Two Sets of GCRA Parameters to Fairly Police PCR and SCR of Connections Passing an ATM Multiplexer, AEÜ, International Journal of Electronics and Communications, 53 (1999) No. 3, 129-134, Urban \& Fischer Verlag.

[8] P. Boyer, F.M. Guillemin, M.J. Servel, J.P. Coudreuse Spacing Cells Protects and Enhances Utilization of ATM Network Links, IEEE Network Magazine, Vol. 6, No. 5, September 1992, pp 38-49.

[9] P. Castelli, A. Forcina, A. Tonietti Dimensioning Criteria for Policing Functions in ATM Networks, INFOCOM ' 92.

[10] V. Catania, G. Ficili, S. Palazzo, D. Panno, A fuzzy decision maker for source traffic control in high speed networks, Proc. 1995 Int. Conf. on Network Protocols (ICNP 95), Tokyo, Nov. 7-10, 1995.

[11] V. Catania, G. Ficili, S. Palazzo, D. Panno, A fuzzy expert system for Usage Parameter Control in ATM networks, Proc. GLOBECOM '95, Singapore, Nov. 13-17, 1995. 
[12] V. Catania, G. Ficili, S. Palazzo, D. Panno, A Comparative Analysis of Fuzzy Versus Conventional Policing Mechanisms for ATM Networks, IEEE/ACM Transactions on Networking, Vol. 4, no. 3, June 1996.

[13] C. Douligeris and G. Develekos, A Fuzzy Logic Approach to Congestion Control in ATM Networks, Proc. IEEE ICC '95, Seattle, WA, June 1995.

[14] J. Heinanen, F. Baker, W. Weiss and J. Wroklawski, Assured Forwarding PHB Group, Internet Draft, January 1999.

[15] F. Huebner, Dimensioning of a Peak Cell Rate Monitor Algorithm Using Discrete-Time Analysis, ITC 14 / J. Labetoulle and J.W. Roberts (Editors).

[16] Z. Jiang, Z. Liu Improved algorithm of usage parameter control in ATM networks, Int. Conf. on Commun. Tech. Proc., ICCT vol. 1, 1996. IEEE, Piscataway, NJ, pp 24-27.

[17] Z. Jiang, Z. Liu Fuzzy leaky bucket for policing mechanism in ATM networks, Neural Networks for Signal Processing. Proc. IEEE Workshop 1996, Piscataway, NJ, pp 510-517.

[18] L. Mason, A. Pelletier, J. Lapointe Toward optimal policing in ATM networks, Computer Communications, vol. 19, no. 3, Mar 1996, pp 194-204.

[19] R. O. Onvural, Asyncronous Transfer Mode Networks: Performance Issues, Norwood, MA: Artech House, 1994.

[20] E. P. Rathgeb, Modeling and Performance Comparison of Policing Mechanisms for ATM Networks, IEEE J. Select. Areas Commun. vol. 9, no. 3, pp. 325-334, Apr. 1991.

[21] J. Sairamesh and N. Shroff, Limitations and pitfalls of leaky bucket, a study with video traffic, Proc. 3rd Int. Conf. Comput. Commun. Networks, Sept. 1994.

[22] A. Tarraf, I. Ibrahim, and T. Saadawi, A novel neural network traffic enforcement mechanism for ATM networks, IEEE J. Select. Areas Commun., vol. 12, no. 6, pp. 1088-1096, Aug. 1994. 